\title{
MicroTrack: An Algorithm for Concurrent Projectome and Microstructure Estimation ${ }^{\star}$
}

\author{
Anthony J. Sherbondy ${ }^{1}$, Matthew C. Rowe ${ }^{2}$, and Daniel C. Alexander ${ }^{2}$ \\ 1 Psychology Department, Stanford University, USA \\ anthony. sherbondy@stanford.edu \\ 2 Computer Science Department, University College of London, UK
}

\begin{abstract}
This paper presents MicroTrack, an algorithm that combines global tractography and direct microstructure estimation using diffusionweighted imaging data. Previous work recovers connectivity via tractography independently from estimating microstructure features, such as axon diameter distribution and density. However, the two estimates have great potential to inform one another given the common assumption that microstructural features remain consistent along fibers. Here we provide a preliminary examination of this hypothesis. We adapt a global tractography algorithm to associate axon diameter with each putative pathway and optimize both the set of pathways and their microstructural parameters to find the best fit of this holistic white-matter model to the MRI data. We demonstrate in simulation that, with a multi-shell HARDI acquisition, this approach not only improves estimates of microstructural parameters over voxel-by-voxel estimation, but provides a solution to long standing problems in tractography. In particular, a simple experiment demonstrates the resolution of the well known ambiguity between crossing and kissing fibers. The results strongly motivate further development of this kind of algorithm for brain connectivity mapping.
\end{abstract}

\section{Introduction}

Diffusion-weighted imaging (DWI) has been used to provide useful markers of brain tissue microstructure in a range of applications including stroke, normal development, aging, and highlighting diseased tissue. Most work uses simple indices, e.g., fractional anisotropy (FA), derived from the diffusion tensor model [7. A major limitation of these simple indices is that they conflate multiple tissue properties. For example, FA correlates with axon density, axon radius distribution, coherence of axon orientations, myelin volume and permeability.

Other previous work $16 / 5 / 4 / 2 \mid 3$ demonstrates the feasibility of measuring specific properties of the tissue directly. The techniques use more sophisticated

\footnotetext{
* AJS sponsored by Defense Advanced Research Projects Agency, Defense Sciences Office (DSO), Program: Systems of Neuromorphic Adaptive Plastic Scalable Electronics (SyNAPSE), Issued by DARPA/CMO under Contract No. HR001109-C-0002; DCA by EPSRC EP/E007748 and the EU CONNECT consortium www.brain-connect.eu
}

T. Jiang et al. (Eds.): MICCAI 2010, Part I, LNCS 6361, pp. 183 190, 2010.

(C) Springer-Verlag Berlin Heidelberg 2010 
models that relate tissue properties like axon density and diameter to the diffusion-weighted MR signal. Early attempts of fitting the model in each voxel 164] have prohibitive data requirements preventing estimation of these parameters on live subjects within a reasonable imaging time. Recently, however, Barazany et al [6] successfully estimate the axon diameter distribution in the brain of a live rat with about $2 \mathrm{~h}$ of imaging time. Moreover, Alexander et al. [2] demonstrate the potential for orientationally invariant estimation of axon density and diameter, even on human imaging systems with around $1 \mathrm{~h}$ acquisition time, through a combination of simplification of the model and careful experiment design. The most recent work 3] shows promising results on data from both fixed monkey brains and live human brains. Orientational invariance [23] offers significant benefits over earlier work [1646, which requires knowledge of the fiber orientation and thus limits parameter maps to structures with that specific orientation. Fiber orientation varies widely in the brain, so orientational invariance is essential for whole brain mapping and enables combination with tractography to study tractwise variation in microstructure. However, the maps of axon diameter and density in [3] are noisy, particularly for live subjects where hardware and acquisition time limitations weaken sensitivity.

Here, we introduce the MicroTrack algorithm that leverages the assumption that microstructure features, e.g., axon radius, myelin volume, and longitudinal diffusivity, remain constant along white matter fascicles. For example, one may estimate an axon diameter distribution that is fixed along the length of a tract such as the optic radiation. The assumption that the axon diameter distribution remains constant along the length of individual fascicles is common in histological studies that characterize tract properties using single cross-section measurements [1] and has been measured to be constant over a range of at least $1 \mathrm{~mm}$ [14. This model provides a powerful geometric constraint that allows us to collapse measurements across multiple voxels to estimate microstructure parameters shared along a single fascicle and potentially improve parameter estimation dramatically.

MicroTrack simultaneously solves for the projectome, i.e., the collection of long-range white matter fascicles projecting between all gray matter locations in the brain [11, and the microstructure tissue parameters of the fascicles within the projectome by fitting a holistic model of the white matter to all the DWI intensities. The white matter model is hierarchical in the sense that it describes both macroscopic features, i.e., pathways joining remote locations, and microscopic features, e.g., the density and diameter of axons in each pathway. This concurrent multiscale modeling is a fundamental augmentation of previous global tractography techniques 121510 and we hypothesize that it offers major benefits over independent estimation of either quantity. First, as mentioned above, pooling data from voxels with similar microstructure improves estimates of axon diameter and density. However, equally important is the potential reduction in false positive connections in the projectome. Specifically, knowledge of the microstructure of individual fascicles resolves ambiguities that occur at, for example, fiber crossing and kissing configurations. At a kissing or crossing, where two 
or more paths are equally likely from their local orientation, the algorithm can pick the path with microstructure most consistent to the rest of the path and reject paths along which significant changes in microstructure occur. This approach offers the first viable and biologically motivated solution to tractography ambiguities like crossing versus kissing fibers.

Section 2 outlines the white matter macro and microstructure models and introduces MicroTrack: the algorithm that simultaneously estimates the projectome and fascicle microstructure properties. Section 3 presents simulation experiments that confirm our central hypotheses of improved microstructure parameter estimation and reduction of false positive projections. We conclude in section 4 with a discussion of limitations and areas for further work.

\section{Method}

This section outlines the MicroTrack algorithm. We start with the forward model of white matter as a set of fascicles linking remote locations each with microstructure that determines the diffusion MR signal from the fascicles in individual voxels. Next, we describe the algorithm that solves the inverse problem or fitting the model to the DWI data. The section goes on to outline the cost function and optimization procedure that enable this fitting.

White Matter Tissue Model. The white matter model captures geometrical architecture of tissue across multiple length scales. Currently, our model considers two length scales: axon bundles or fascicles, with cross-sectional radius on the order of hundreds of microns, and axons, with cross-sectional radius on the order of a micron. The fascicles are simple tubular structures that are defined by a sequence of backbone points that extend between distant gray matter locations and a single cross-sectional radius $(R)$ for the entire fascicle. At the sub-fascicle scale, the composition of the fascicle structure is characterized by several parameters: local orientation $\mathbf{n}$, average axon radius $r$, fraction of fascicle cross-section filled with axons $f$, intra-axonal diffusivity $d$, and extra-axonal diffusivity, which we assume is also $d$, but that tortuosity around the axons reduces the perpendicular apparent diffusivity to $d_{p}$. Orientation is the only parameter that varies along the fascicle and all other microstructural parameters are fixed within each.

The tissue model predicts MR attenuation within a voxel composed of a mixture of fascicles and non-fascicle volume by dividing each voxel into compartments that contain only one tissue type in a similar way to Close et al. 8. Specifically, we divide the voxel grid into a much higher resolution subvoxel grid, typically $3 \times 3 \times 3$ subvoxels per image voxel. Any subvoxels within fascicles are considered fascicle compartments and the rest contribute cerebral spinal fluid (CSF). Occasionally, fascicles overlap on a subvoxel; the closest fascicle determines the microstructure and orientation of these subvoxels' tissue.

Signal Model. The simplified CHARMED model in 23. provides the signal in subvoxels within fascicles. In this model, the normalized MR signal is 


$$
A\left(\mathbf{G}, \Delta, \delta ; \mathbf{n}, r, f, d, d_{p}\right)=f A_{r}(\mathbf{G}, \Delta, \delta ; \mathbf{n}, r, f, d)+(1-f) A_{h}\left(\mathbf{G}, \Delta, \delta ; \mathbf{n}, d, d_{p}\right),
$$

where $\mathrm{f}$ is the volume fraction of the restricted water inside axons, which has normalized signal $A_{r}$, and $A_{h}$ is the signal from the hindered water outside but amongst the axons; $\mathbf{G}$ is the gradient vector, $\delta$ is the width of the gradient pulses in the pulsed-gradient spin-echo sequence, and $\Delta$ is their separation. The intracellular signal, $A_{r}$, is the product of attenuated signals from the components of $\mathbf{G}$ parallel and perpendicular to the fiber direction [5]. We assume Gaussian displacements with diffusivity $d$ parallel to the fiber and use the Gaussian Phase Distribution approximation [13] of the signal from spins with diffusivity $d$ restricted within a cylinder of radius $r$ [18] for the perpendicular signal. The signal, $A_{h}$, from the hindered compartment comes from the diffusion tensor model with principal eigenvector $\mathbf{n}$ and eigenvalue $d$ and minor eigenvalues $d_{p}$. The signal model for CSF subvoxels assumes isotropic Gaussian displacements with diffusivity $d_{i s o}$. The final set of signals for each whole voxel is the average of each corresponding measurement over all the subvoxels within.

Algorithm Overview. The MicroTrack algorithm follows the general optimization procedure of a recent projectome identification algorithm [15]. MicroTrack takes as input a collection of $N_{C}$ fascicle candidates $(C)$ and outputs a vector of fascicle radii $\left(\bar{R}=\left[R_{1}, R_{2}, R_{3}, \ldots, R_{N_{C}}\right]\right)$, but it also outputs a vector of microstructure parameters $\left(\bar{r}=\left[r_{1}, r_{2}, r_{3}, \ldots, r_{N_{C}}\right]\right)$. The algorithm searches over configurations of $\bar{r}$ and $\bar{a}$ to minimize the error function $E(\bar{R}, \bar{r})$ that balances fitting the MRI data with volumetric constraints that penalize fascicle overlap. Similar to the previous projectome identification algorithm, $\bar{R}$ is binarized so that each fascicle may either be included in the optimal projectome, $R_{i}>0$, or excluded, $R_{i}=0$. Unlike the previous algorithm, MicroTrack also searches over a discrete set of $r_{i}$ values that best characterize the fascicle candidate.

Cost Function. MicroTrack searches over configurations of $\bar{R}$ and $\bar{r}$ in order to select the setting that minimizes the global error, $E=\lambda E_{1}-(1-\lambda) E_{2}$. $E_{1}$ measures the difference between the predicted and observed MRI signal attenuation, and, $E_{2}$ measures the amount of overlap between the fascicle structures. The parameter $\lambda$ balances the effects of $E_{1}$ and $E_{2}$.

The data fitting error, $E_{1}$, is a straightforward square error metric summed over the number of voxels $(V)$ and number of MR measurements per voxel $(K)$,

$$
E_{1}=\sum_{v=1}^{V} \sum_{k=1}^{K} \frac{\left(\hat{A}_{k, v}-A_{k, v}\right)^{2}}{\sigma^{2}},
$$

where $A_{k, v}$ is the predicted DWI signal, $\hat{A}_{k, v}$ is actual MRI and $\sigma$ is the standard deviation of the measurements, which is fixed for the entire volume.

The fascicle volume overlap error, $E_{2}$, simply reports the volume of fascicles that overlap, i.e., violate physical integrity of fascicles by occupying the same space. The units of $E_{2}$ are in fractions of the voxel volume. For example, if 
a subvoxel is overlapped by two fascicles than a penalty of $1 / N_{s}$, where $N_{s}$ is the number of subvoxels, is added to $E_{2}$ for that voxel and if three fascicles overlap the subvoxel the penalty is $2 / N_{s}$ and so on.

Fascicle Candidates. MicroTrack precomputes a large set, $C$, of candidate fascicles using either individual or combinations of standard local deterministic and probabilistic tracking algorithms (Camino, FSL, DTIStudio, MRTRIX, TrackVis). For this work, the candidate set was created using the Camino software [9, which offers both deterministic and probabilistic tractography with HARDI measurement schemes. Other than the endpoint criteria, all default parameter values are used and seed points are randomly selected within the entire volume.

Optimization Strategy. The search space of all possible white matter configurations is very high-dimensional and the cost function has many local minima. Here we use a stochastic optimization algorithm, differential evolution (DE) [17, which we have found to outperform a variety of other off the shelf global optimization algorithms. DE fits in the class of genetic evolution algorithms that search over a parameter space by evolving individuals (parameter settings) amongst a community (several parameter settings) based on the individual's fitness (error function). The stochastic/genetic search is advantageous for our problem as multiple individuals may fit disparate parts of the white matter volume well and combine to provide the benefits of both. The complexity of the problem prevents guaranteed convergence on the global minimum, in practice we find that the following parameter settings: (generations $=1600$, populations $=40, \mathrm{CR}=0.9, \mathrm{~F}=[0.5,0.8]$, resets $\left.=8, r_{i} \in[1,5,10] \mu m, R_{i} \in[0,0.2] \mathrm{mm}\right)$ produce good, if suboptimal, solutions in a reasonable time ( 2 hours on 82.3 $\mathrm{GHz}$ CPUs).

\section{Results}

The intention of the experiments is to test the core hypothesis that simultaneous estimation of the projectome and microstructure is advantageous over estimation of either independently. We thus construct several simple synthetic data sets specifically to enable such a test. The synthetic data was produced using a modification of the Numerical Fiber Generator (NFG) software 8. The NFG software offers an efficient way of producing crossing structures with complex intersections. For the synthetic data used here, we replace the tensor model in the original NFG software with the two compartment model in equation 1. Each strand of the strand structure is assigned an individual axon radius, in our examples 1,5 or $10 \mu \mathrm{m}$. The other parameters have fixed values of $d=1.7 \times 10^{-9}$ $\mathrm{m}^{2} / \mathrm{s}, d_{p}=1.2 \times 10^{-9} \mathrm{~m}^{2} / \mathrm{s}, d_{i s o}=3.0 \times 10^{-9} \mathrm{~m}^{2} / \mathrm{s}, f=0.7$. The system adds Rician noise so that the signal to noise ratio at $b=0$ is 20 .

The imaging protocol we simulate is a multi-shell HARDI acquisition obtained from the experiment-design optimization in 2, which optimizes sensitivity to axon diameter. The three shells have $b=2242(|\mathbf{G}|=200 \mathrm{mT} / \mathrm{m}, \Delta=14.5 \mathrm{~ms}$, $\delta=8 \mathrm{~ms}), 3791(|\mathbf{G}|=121 \mathrm{mT} / \mathrm{m}, \Delta=23.4 \mathrm{~ms}, \delta=13.8 \mathrm{~ms})$, and $11329 \mathrm{~s} / \mathrm{mm}^{2}$ 

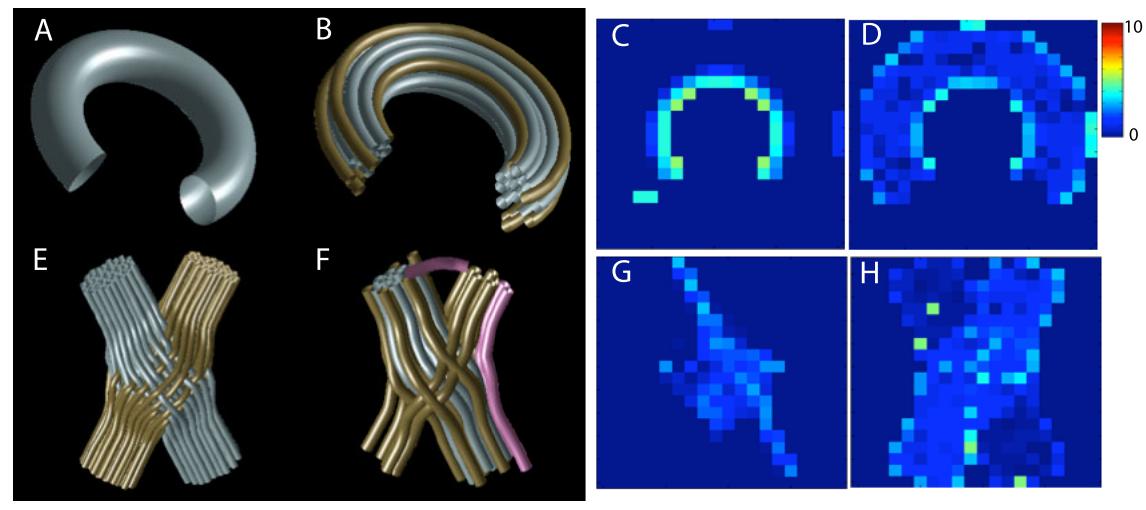

Fig. 1. MicroTrack improves microstructure estimation accuracy. Torus (A) and oblique crossing (E) data are simulated and reconstructed with MicroTrack (B,F). Error in mean axon radius estimation for a central slice (C,G) reduces error compared with the estimation using a typical voxel-by-voxel algorithm $(\mathrm{D}, \mathrm{H})$. Fibers are labeled based on mean axon radius as either 1 (blue), 5 (brown), or $10 \mu m$ (purple). Pink fibers represent false positive connections. Error bar is in units of $\mu \mathrm{m}$.

$(|\mathbf{G}|=200 \mathrm{mT} / \mathrm{m}, \Delta=21.8 \mathrm{~ms}, \delta=15.3 \mathrm{~ms})$, and 100,105 and 84 gradient directions, respectively. The protocol also includes $65 b=0$ measurements for a total of 364 images.

Improving Microstructure Estimation. We compare MicroTrack estimates of white matter fraction and average axon radius within each voxel to estimates from the grid search and maximum likelihood fit steps of the fitting algorithm in [3] a simple gradient-descent fit of the tissue parameters independently for each voxel. The precise model we fit is as in equation 1, but with an extra CSF compartment using the same CSF signal model as the simulation. The comparison is carried out on two synthetic data sets: cut torus (Figure 1]A) and oblique crossing (Figure 1E). The MicroTrack estimates reduce the error over most voxels in both synthetic experiments and, in particular, are more accurate in the voxels where the fiber orientation distributions are multi-modal.

Reducing Tracking Ambiguities. In order to focus in on the hypothesis that concurrent microstructure and projectome estimation can improve the connectivity results over standard tracking techniques, we created two synthetic volumes that demonstrate the well-known kissing versus crossing ambiguity (Figure 2A and D). The synthetic volume was carefully constructed so that the white matter in the oblique crossing configuration overlaps the white matter volume in the kissing configuration as much as possible. The result is that many voxels within the two volumes contain nearly identical fiber orientation distributions, despite a fundamental difference in connectivity structure. To further focus the experiment, the fascicle candidate set was derived from the gold standard fascicles used to create the synthetic data. This ensures that the candidate fascicle set, 


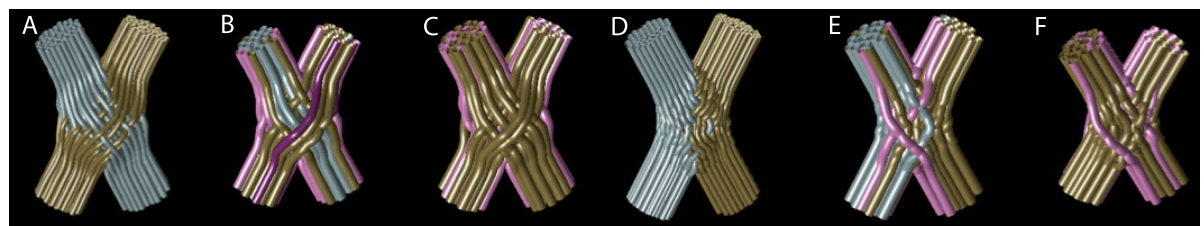

Fig. 2. MicroTrack addresses orientation ambiguity. Classic crossing (A-C) and kissing (D-F) data are simulated (A,D), reconstructed with MicroTrack (B,E) and reconstructed MicroTrack, but with concurrent microstructure estimation disabled (C,F). Fibers are labeled based on mean axon radius as either 1 (blue), 5 (brown), or $10 \mu \mathrm{m}$ (purple). Pink fibers represent false positive connections.

$C$, contains equal numbers of candidate fibers that follow the crossing and kissing configurations, which maximizes the ambiguity for the algorithm to resolve. If we solve for the best fitting projectome without solving for microstructure parameters the false positive rate is $40 \pm 2 \%$ (Figure $2 \mathrm{C}$ and $\mathrm{F}$ ). When we simultaneously fit the microstructure parameters the false positive rate is reduced by nearly 3 fold to $14 \pm 1 \%$ false positives (Figure $2 \mathrm{~B}$ and $\mathrm{E}$ ).

\section{Conclusion}

This paper demonstrates the first algorithm to constrain the solution of microstructural tissue properties with fascicle geometry estimates within an identified projectome. The algorithm is also the first to use microstructural tissue properties to determine the fascicle geometry. Despite the tremendous computational complexity of solving for parameters in this very high dimensional space, we demonstrate the feasibility of the algorithm. The simple simulation experiments demonstrate the advantages of employing such a detailed model of white matter tissue that not only incorporates global fascicular projection geometry, but also geometrical details of tissue microstructure. In particular, we demonstrate the potential to resolve crossing and kissing configurations. Further work will refine the model for use with real brain tissue and test the hypothesis on well-known anatomical crossings and kissings.

\section{References}

1. Aboitiz, F., Scheibel, A.B., Fisher, R.S., Zaidel, E.: Fiber composition of the human corpus callosum. Brain Research 598, 143-153 (1992)

2. Alexander, D.C.: A general framework for experiment design in diffusion MRI and its application in measuring direct tissue-microstructure features. Magnetic Resonance in Medicine 60, 439-448 (2008)

3. Alexander, D.C., Hubbard, P.L., Hall, M.G., Moore, E.A., Ptito, M., Parker, G.J.M., Dyrby, T.D.: Orientationally invariant indices of axon diameter and density from diffusion MRI. NeuroImage (2010) (in press) 
4. Assaf, Y., Blumenfeld-Katzir, T., Yovel, Y., Basser, P.J.: AxCaliber: a method for measuring axon diameter distribution from diffusion MRI. Magnetic Resonance in Medicine 59, 1347-1354 (2008)

5. Assaf, Y., Freidlin, R.Z., Rohde, G.K., Basser, P.J.: New modeling and experimental framework to characterize hindered and restricted water diffusion in brain white matter. Magnetic Resonance in Medicine 52, 965-978 (2004)

6. Barazany, D., Basser, P.J., Assaf, Y.: In-vivo measurement of the axon diameter distribution in the corpus callosum of a rat brain. Brain 132, 1210-1220 (2009)

7. Basser, P.J., Pierpaoli, C.: Microstructural and physiological features of tissues elucidated by quantitative diffusion tensor MRI. Journal of Magnetic Resonance Series B 111, 209-219 (1996)

8. Close, T.G., Tournier, J.-D., Calamante, F., Johnston, L.A., Mareels, I., Connelly, A.: A software tool to generate simulated white matter structures for the assessment of fibre-tracking algorithms. NeuroImage 47(4), 1288-1300 (2009)

9. Cook, P.A., Bai, Y., Nedjati-Gilani, S., Seunarine, K.K., Hall, M.G., Parker, G.J.M., Alexander, D.C.: Camino: Open-source diffusion-MRI reconstruction and processing. In: Proc. 14th Annual Meeting of the ISMRM, Berlin (2006)

10. Fillard, P., Poupon, C., Mangin, J.-F.: A novel global tractography algorithm based on an adaptive spin glass model. In: Yang, G.-Z., Hawkes, D., Rueckert, D., Noble, A., Taylor, C. (eds.) MICCAI 2009. LNCS, vol. 5761, pp. 927-934. Springer, Heidelberg (2009)

11. Kasthuri, N., Lichtman, J.W.: The rise of the 'projectome'. Nat. Methods 4(4), 307-308 (2007)

12. Kreher, B.W., Mader, I., Kiselev, V.G.: Gibbs tracking: A novel approach for the reconstruction of neuronal pathways. Magnetic Resonance in Medicine 60, 953-963 (2008)

13. Murday, J.S., Cotts, R.M.: Self-diffusion coefficient of liquid lithium. Journal of Chemical Physics 48, 4938-4945 (1968)

14. Ong, H.H., Wright, A.C., Wehrli, S.L., Souza, A., Schwartz, E.D., Hwang, S.N., Wehrli, F.W.: Indirect measurement of regional axon diameter in excised mouse spinal cord with q-space imaging: Simulation and experimental studies. NeuroImage 40(4), 1619-1632 (2008)

15. Sherbondy, A.J., Dougherty, R.F., Ananthanarayanan, R., Modha, D.S., Wandell, B.A.: Think global, act local; projectome estimation with BlueMatter. In: Yang, G.-Z., Hawkes, D., Rueckert, D., Noble, A., Taylor, C. (eds.) MICCAI 2009. LNCS, vol. 5761, pp. 861-868. Springer, Heidelberg (2009)

16. Stanisz, G.J., Szafer, A., Wright, G.A., Henkelman, M.: An analytical model of restricted diffusion in bovine optic nerve. Magnetic Resonance in Medicine 37, 103-111 (1997)

17. Storn, R., Price, K.: Differential evolution a simple and efficient heuristic for global optimization over continuous spaces. Journal of Global Optimization 11, 341-359 (1997)

18. Van Gelderen, P., DesPres, D., van Zijl, P.C.M., Moonen, C.T.W.: Evaluation of restricted diffusion in cylinders. Phosphocreatine in rabbit leg muscle. Journal of Magnetic Resonance Series B 103, 255-260 (1994) 